Ana Daniela Cabrera-Padrón; Darwin Gabriel García-Herrera; Juan Carlos Erazo-Álvarez Cecilia Ivonne Narváez-Zurita

http://dx.doi.org/10.35381/r.k.v5i5.1045

\title{
Redes de apoyo mediante la trilogía 3Cs para fortalecer el proceso de enseñanza aprendizaje
}

\section{Support networks through the $3 \mathrm{Cs}$ trilogy to strengthen the teaching-learning process}

\author{
Ana Daniela Cabrera-Padrón \\ anadaniela.cabrera@est.ucacue.edu.ec \\ Universidad Católica de Cuenca, Azogues \\ Ecuador \\ https://orcid.org/0000-0003-0728-0351 \\ Darwin Gabriel García-Herrera \\ dggarciah@ucacue.edu.ec \\ Universidad Católica de Cuenca, Azogues \\ Ecuador \\ https://orcid.org/0000-0001-6813-8100 \\ Juan Carlos Erazo-Álvarez \\ jcerazo@ucacue.edu.ec \\ Universidad Católica de Cuenca, Cuenca \\ Ecuador \\ https://orcid.org/0000-0001-6480-2270 \\ Cecilia Ivonne Narváez-Zurita \\ inarvaez@ucacue.edu.ec \\ Universidad Católica de Cuenca, Cuenca \\ Ecuador \\ https://orcid.org/0000-0002-7437-9880
}

Recibido: 15 de septiembre de 2020

Revisado: 19 de octubre de 2020

Aprobado: 15 de noviembre de 2020

Publicado: 01 de diciembre de 2020 
Revista Arbitrada Interdisciplinaria KOINONIA

Año 2020. Vol V. N5. Especial II: Educación

Hecho el depósito de Ley: FA2016000010

ISSN: 2542-3088

FUNDACIÓN KOINONIA (F.K). Santa Ana de Coro. Venezuela.

Ana Daniela Cabrera-Padrón; Darwin Gabriel García-Herrera; Juan Carlos Erazo-Álvarez Cecilia Ivonne Narváez-Zurita

\title{
RESUMEN
}

El objetivo fue analizar la factibilidad de implementar redes de apoyo en la Unidad Educativa del Milenio Paiguara de la ciudad de Gualaceo - Ecuador, para fortalecer el proceso de enseñanza aprendizaje, mediante el intercambio de conocimientos y experiencias trabajados colaborativamente. Metodológicamente se trabajó con un enfoque mixto de prevalencia descriptiva, recopilandose datos cuantitativos. Es importante señalar que para mejorar la calidad en el proceso de enseñanza - aprendizaje dentro de un contexto educativo, todos sus integrantes deben estar comprometidos con la institución educativa, esto se logra con la debida motivación y consensos por parte de las autoridades del establecimiento, además deben construir participativamente objetivos a corto, mediano y largo plazo, solo de este modo se podrán aunar esfuerzos, proyectar propuestas y trabajar colaborativamente para alcanzar la meta común planteada.

Descriptores: Experimento educacional; innovación educacional; innovación pedagógica. (Palabras tomadas del Tesauro UNESCO).

\begin{abstract}
The objective was to analyze the feasibility of implementing support networks in the Paiguara Millennium Educational Unit of the city of Gualaceo - Ecuador, to strengthen the teaching-learning process, through the exchange of knowledge and experiences worked collaboratively. Methodologically, a mixed approach of descriptive prevalence was used, collecting quantitative data. It is important to point out that to improve the quality of the teaching-learning process within an educational context, all its members must be committed to the educational institution, this is achieved with the proper motivation and consensus on the part of the establishment authorities, in addition They must build short, medium and long-term objectives in a participatory manner, only in this way can they join forces, project proposals and work collaboratively to achieve the common goal set.
\end{abstract}

Descriptors: Educational experiments; educational innovations; teaching method innovations. (Words taken from the UNESCO Thesaurus). 


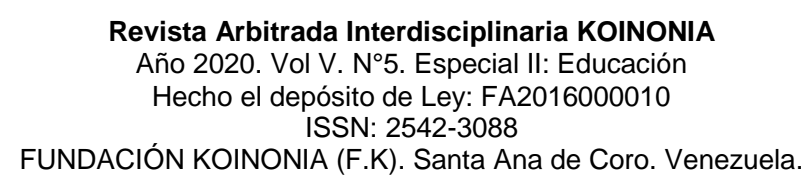

Ana Daniela Cabrera-Padrón; Darwin Gabriel García-Herrera; Juan Carlos Erazo-Álvarez Cecilia Ivonne Narváez-Zurita

\section{INTRODUCCIÓN}

Es sabido que la educación es un factor imprescindible en el desarrollo de un país, lamentablemente la actual crisis sanitaria atenta directamente contra la calidad educativa por factores como acceso a internet, conocimiento y uso de las TIC; y la predisposición, motivación y capacitación de los docentes para enfrentar este nuevo desafío. El tema que aquí nos interesa es; ¿qué podemos hacer como docentes para fortalecer el proceso de enseñanza aprendizaje durante la época de pandemia?

Para lograr un cambio en el sistema educativo es importante según (Feixas, 2004), empezar por cambiar el modelo preconcebido tradicional que tienen los docentes, sobre como aprenden los estudiantes. Para lograr esto es importante la predisposición al cambio de cada profesional de la educación y lo que estamos dispuestos a aportar.

Esto no quiere decir que todo lo que se ha hecho hasta aquí en cuanto a educación está mal, ya que para (Murillo \& Krichesky, 2014), el mejoramiento de la práctica educativa está basado en las experiencias cotidianas de los docentes, en ultimas, para que esto rinda frutos se requiere de reflexiones críticas, socializadas por parte de cada integrante de la red de apoyo en las instituciones educativas. En esta misma línea (Fullan \& Hargreaves, 1997), manifiestan que, intervenir o reflexionar sobre la práctica educativa o el desempeño docente dentro y fuera de las aulas de clase nos permitirá realizar una autoevaluación con miras a mejorar nuestra destreza profesional.

En consecuencia, la práctica docente es un cúmulo de experiencias de aula complejas, lamentablemente en muchas ocasiones nos encontramos con docentes que se cierran ante sugerencias o simplemente no aceptan la retroalimentación luego del acompañamiento por parte de las autoridades, esto les lleva a trabajar de manera individual. Sin embargo, (Vaillant, 2016), invita a reflexionar que cuando se plantean problemáticas de manera colaborativa, aumentan las posibilidades de encontrar más y mejores herramientas que favorezcan al proceso de enseñanza aprendizaje. El mismo autor manifiesta que trabajo colaborativo permiten que un grupo de docentes estudien, 


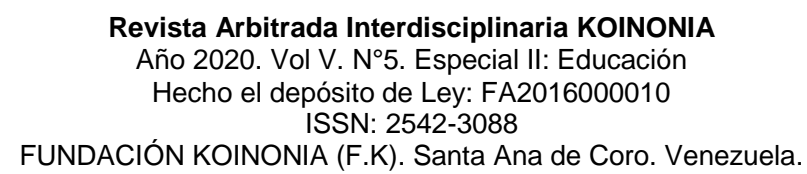

Ana Daniela Cabrera-Padrón; Darwin Gabriel García-Herrera; Juan Carlos Erazo-Álvarez Cecilia Ivonne Narváez-Zurita

intercambien experiencias, reflexionen e investiguen juntos acerca de sus prácticas educativas, en un contexto institucional y social determinado.

La cooperación entre docentes es una necesidad en la actualidad para fortalecer las prácticas educativas y hacer frente a una nueva realidad, el trabajo colaborativo no es aplicable únicamente entre estudiantes, sino se puede implementar entre el círculo de docentes en el contexto educativo. Holísticamente para (Stoll, et al., 2006), las redes de apoyo entre docentes se pueden definir como un grupo de profesionales en educación que comparten y cuestionan críticamente su práctica, de manera continua, reflexiva y colaborativa, con una orientación al aprendizaje de los alumnos y el crecimiento profesional.

Mientras que para (Cochran-smith \& Lytle, 2006), la integración de redes de apoyo entre docentes con diferentes años de edad y experiencia permitirá integrar nuevos conocimientos sobre la práctica docente, reflexionando sobre lo que se ha hecho y lo que falta por hacer, es decir consolidar experiencias positivas que permitirán mejorar la práctica educativa y descartar experiencias que no permiten alcanzar aprendizajes significativos.

Además de las redes de apoyo, otras de las estrategias para mejorar la calidad educativa según (Ponce-Ceballos, et al., 2018), es implementar proyectos de mentorización aprovechando recursos humanos disponibles dentro de las instituciones educativas como; docentes con experiencia, con actualizaciones recientes o con títulos de cuarto nivel, esto permitirá fomentar el compañerismo, mejorar la seguridad y disciplina en el aula y adquirir nuevos conocimientos.

El trabajo colaborativo entre los docentes de una institución educativa es sin duda una de las mejores estrategias para avivar la práctica docente, hemos analizado que implica la retroalimentación de experiencias, conocimientos y motivación sin duda alguna, para Aparicio-Molina \& Sepúlveda-López, 2019), además de todos estos beneficios permite vencer la rutina en la jornada laboral, la enajenación del rol como docentes y el desgaste profesional. 
Ana Daniela Cabrera-Padrón; Darwin Gabriel García-Herrera; Juan Carlos Erazo-Álvarez Cecilia Ivonne Narváez-Zurita

Amparados en estos criterios varios países se han organizado para trabajar cooperativamente formando redes de apoyo para el mejoramiento del nivel educativo en cado uno de sus territorios como, por ejemplo:

El (Ministerio de Educación del Ecuador en el año 2019), plantea un programa: "Redes de aprendizaje, conformación e implementación de redes de aprendizaje en instituciones educativas de sostenimiento fiscal, fisco misional, municipal y particular que ofertan el nivel de educación inicial y preparatoria, con el objetivo elevar la calidad de la gestión y la atención educativa en el nivel de Educación Inicial y la articulación con El objetivo fue analizar la factibilidad de implementar redes de apoyo en la Unidad Educativa del Milenio Paiguara de la ciudad de Gualaceo - Ecuador, para fortalecer el proceso de enseñanza aprendizaje, mediante el intercambio de conocimientos y experiencias trabajados colaborativamente.

\section{Referencial teórico}

En la última década se ha puesto un especial énfasis al trabajo colaborativo entre docentes, por lo que al realizar una búsqueda sobre este particular encontramos abundante información sobre la importancia del trabajo colaborativo y su aplicación dentro de la formación de redes de apoyo, pero los estudios se enfocan únicamente en temas puntuales como educación inicial e inclusión, pero sorprende comprobar que existe escasa información sobre la formación de redes de apoyo para el fortalecimiento de la práctica docente. Se describe a continuación trabajos investigativos relacionados al tema planteado que han sido realizados en otros países en los 3 últimos años.

a. Fortaleciendo el trabajo colaborativo con las redes de apoyo de los establecimientos educativos.

b. Redes de apoyo y colaboración para la mejora de la educación inclusiva.

El primer artículo de título: "Fortaleciendo el trabajo colaborativo con las redes de apoyo de los establecimientos educativos", fue llevado a cabo por estudiantes de Parvularia de la facultad de Educación de la Universidad Alberto Hurtado en Chile y publicado en 2017. 


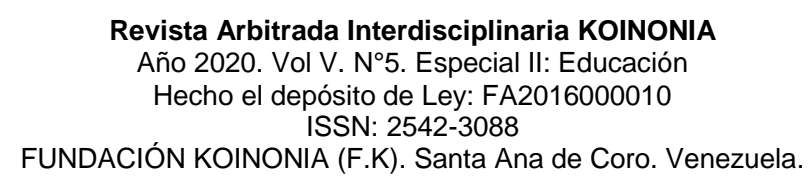

Ana Daniela Cabrera-Padrón; Darwin Gabriel García-Herrera; Juan Carlos Erazo-Álvarez Cecilia Ivonne Narváez-Zurita

Para (Araos \& Álvarez, 2017), trabajar vinculados con otros miembros de la comunidad educativa permite intercambiar experiencias y generar vínculos que nos conduzcan a formar un entorno de apoyo accesible. Para los autores el documento muestra varias propuestas para el fortalecimiento del trabajo colaborativo ente los docentes de Inicial mediante el uso de redes de apoyo entre varios establecimientos educativos.

La propuesta de este artículo es contribuir a la formación inicial docente, para que sea un individuo interactivo, participativo, comprometido, no solo en la enseñanza de conocimientos de los niños y las niñas, sino como un docente integrador de experiencias desde sus individualidades propias para nutrirlos diferentes ámbitos de la labor educativa. El segundo artículo de tema: "Redes de apoyo y colaboración para la mejora de la educación inclusiva", es un trabajo investigativo de tipo evaluativo, se desarrolló en Murcia España y fue publicado en 2018, para sus autores (Sánchez, et al., 2018), está fundamentado en el derecho de toda persona a recibir una educación equitativa e inclusiva, sostienen que es necesario la reflexión y la continua valoración de manera colaborativa para alcanzar las metas planteadas. El propósito de este trabajo investigativo es promover la inclusión dentro de la enseñanza obligatoria. En el artículo se puede apreciar las ventajas que se obtienen al formar una red de apoyo entre instituciones educativas de una misma localidad.

Pues bien, luego de obtener información de artículos científicos afines, para el entender el contexto de nuestra, se necesita entender la situación actual de confinamiento que vive el mundo ocasionada por el Covid-19, que ha generado un fuerte impacto en los procesos de enseñanza aprendizaje, la implementación la modalidad virtual en las clases y sumada la fuerte crisis económica, han generado una brecha entre los estudiantes con acceso a internet y con los que no disponen de recursos tecnológicos.

En este contexto surge la necesidad del fortalecimiento docente en el manejo de herramientas digitales y estrategias metodológicas para fortalecer el proceso de enseñanza aprendizaje, buscando los recursos y las herramientas necesarias para atender a las individualidades de cada grupo de estudiantes. Por otra parte, al hablar de 


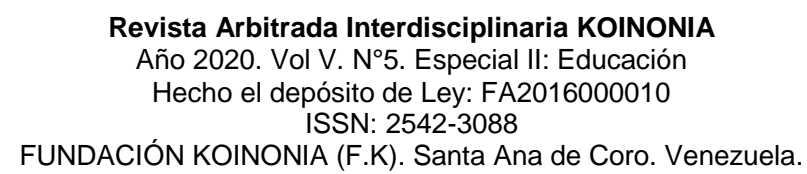

Ana Daniela Cabrera-Padrón; Darwin Gabriel García-Herrera; Juan Carlos Erazo-Álvarez Cecilia Ivonne Narváez-Zurita

aislamiento social, no quiere decir que en esta época de confinamiento debemos descuidar el trabajo colaborativo, al contrario, debemos buscar modalidades y recursos digitales que nos permitan el fortalecimiento de la gestión docente con miras a la calidad educativa.

\section{Trabajo colaborativo}

La relación entre integrantes de una comunidad educativa, con un objetivo en común como es mejorar la calidad educativa, va más allá de sonreír y ser cordiales, tiene una implicación mucho más grande, como aunar esfuerzos, sumar propuestas y formar vínculos que perduren en el tiempo, esto permitirá un mayor impacto en los logros planteados con un menor desgaste físico y mental.

Una de las estrategias más actuales para el crecimiento profesional docente, es la formación de redes de apoyo entre los miembros de la comunidad educativa, más, no podemos hablar de formación de redes de apoyo docente y mucho menos de fortalecimiento entre pares sin antes mencionar el trabajo colaborativo, según manifiesta (Montse-Guitert \& Ferran-Giménez, 2000), el trabajo colaborativo es una sucesión de saberes en el que cada individuo aprende más de lo haría individualmente, gracias a la interacción colectiva con el aporte de cada uno de los miembros del grupo. Para que el trabajo colaborativo rinda frutos es importante una comunicación bidireccional con respeto a los diferentes puntos de vista y criterios que permitan alcanzar un proceso de construcción y retroalimentación de conocimientos.

Dicho de otro modo, el trabajo colaborativo para (Ramírez-Iñiguez, 2017), implica la colaboración en el contexto educativo que permite reflexionar a docentes y estudiantes a plantearse un objetivo en común a lograr sumando experiencias, reflexiones, opiniones y competencias que deben ser aplicadas en la práctica para que rindan los frutos esperados. Entre los puntos importantes del aprendizaje colaborativo, esta que permite la socialización e intercambio de ideas entre los integrantes del grupo, fomenta el respeto y tolerancia a la diversidad de ideas, pensamientos y opiniones a más de fortalecer la 


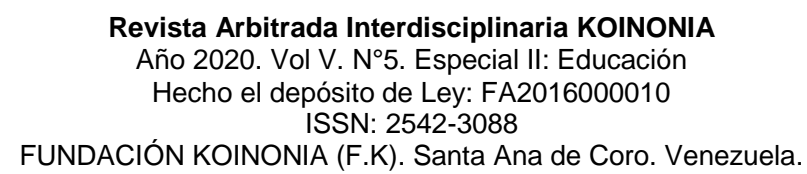

Ana Daniela Cabrera-Padrón; Darwin Gabriel García-Herrera; Juan Carlos Erazo-Álvarez Cecilia Ivonne Narváez-Zurita

práctica docente. Para (Podestá, 2014), es imprescindible la organización y el planteamiento claro de normas dentro del trabajo colaborativo, el autor sintetiza lo elemental dentro del trabajo en equipo:

1. La distribución de responsabilidades entre los integrantes, además de la programación de medios de comunicación, espacios y tiempos en los que se llevará a cabo el compartir de la información.

2. La comunicación enmarcada en el respeto es la clave para el éxito den el cumplimiento de las metas planteadas.

3. Uso de herramientas digitales para la comunicación como; foros, chats, video llamadas, que resulta interesante en la época actual por la que atravesamos.

4. La planificación y cumplimiento de actividades debe ser consensuada y compartida.

Una de las ventajas en la actualidad para el trabajo colaborativo es la gran variedad y recursos digitales gratuitos que se encuentran a disposición de todos los docentes, únicamente se requiere una actitud investigativa para innovar estos procesos.

\section{Redes de apoyo}

Para (Muijs \& Rumyantseva, 2014), uno de los importantes progresos en cuanto a educación se refiere en los últimos años es la formación de redes de apoyo entre docentes, escuelas, universidades, que pueden llegar a ser aplicados inclusos en diferentes contextos sociales, esta cultura de trabajo colaborativo permitirá alcanzar más rápido y de mejor manera los objetivos planteados.

Al hablar de redes de colaboración o redes de apoyo educativo los autores (Sánchez, et al., 2018), lo han definido como agrupaciones de individuos con una meta clara y alcanzable dentro del contexto educativo, está basada en la colaboración socioeducativa de cada uno de los integrantes, para que los beneficios de esta estrategia sean aplicados a la práctica es importante definir acuerdos y compromisos dentro del grupo de trabajo. 


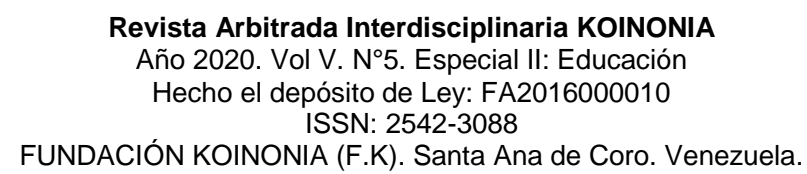

Ana Daniela Cabrera-Padrón; Darwin Gabriel García-Herrera; Juan Carlos Erazo-Álvarez Cecilia Ivonne Narváez-Zurita

El (Ministerio de Educación del Ecuador, 2019), en la guía de conformación e implementación de redes de aprendizaje en instituciones educativas de sostenimiento fiscal, fisco misional, municipal y particular que ofertan el nivel de educación inicial y preparatoria, plantea tres tipos de aprendizaje:

1. Aprendizaje colaborativo con un enfoque pedagógico en el que se contempla aspectos tales como: Currículo, Modelo Pedagógico, Interactividad, Didácticas y la Sociedad del Conocimiento.

2. Aprendizaje cooperativo basado en la parte tecnológica, en los que se integran: Criterios técnicos, logística, producción de materiales, interactividad y soporte de redes y diseño de medios.

3. Aprendizaje activo con un enfoque comunicativo integrando aspectos como: Criterios y procedimientos de mediación, sensibilización y desarrollo de la Estrategia de Comunicación.

De esto modo se pretende fortalecer la educación inicial y preparatoria como prioridad, ya que son los años de educación que cimientan la preparación académica de los estudiantes. Dentro los beneficios que ofrecen la conformación de redes tenemos:

1. Mantener un dialogo asertivo entre los integrantes de la red, esto se logra escuchando respetuosamente opiniones, criterios y puntos de vista diferentes al que se propone.

2. Cambiar la mentalidad sobre enfoques educativos y puntos de vista sobre la práctica docente en base a la reflexión de las ventajas y desventajas que se encuentran en las diferentes propuestas.

3. Implementar el análisis crítico, el pensamiento innovador y el intercambio de experiencias, para el mejoramiento de la práctica docente mediante el ejercicio creativo.

4. Sustituir el celo profesional y el individualismo por el compañerismo y el trabajo colaborativo, esto se logra analizando los beneficios y aprendizajes que se obtienen trabajando en equipo. 


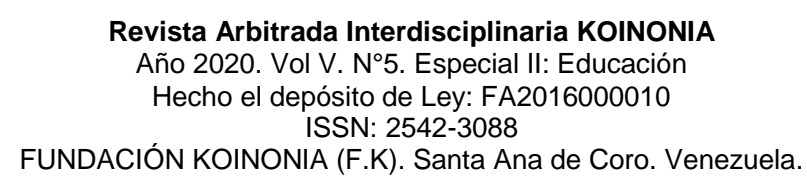

Ana Daniela Cabrera-Padrón; Darwin Gabriel García-Herrera; Juan Carlos Erazo-Álvarez Cecilia Ivonne Narváez-Zurita

5. Realizar una autoevaluación sobre la práctica docente, con mentalidad abierta en post de buscar herramientas que nos permitan conocer la individualidad de cada estudiante, detectar los problemas presentados dentro y fuera del aula y nos lleven al mejoramiento del proceso de enseñanza aprendizaje.

6. Relacionar las experiencias educativas propias con la de los demás y de ese modo construir un marco conceptual referencial para la innovación y contextualización de experiencias como punto de partida para el cambio.

7. Generar espacios de dialogo e intercambio entre miembros de la comunidad educativa y porque no, entre instituciones educativas, permitiendo el progreso de las potencialidades personales e institucionales y la retroalimentación, en los diferentes aspectos educativos.

Con el análisis realizado hasta aquí, podemos citar que la formación de redes de apoyo entre docentes permite el fortalecimiento de la práctica educativa mediante el intercambio de experiencias, en temas como: fortalecimiento de niveles educativos, inclusión, uso y aplicación de herramientas digitales, potencialización de áreas de aprendizaje y uso de estrategias metodológicas activas.

La formación de redes de aprendizaje que permitan el fortalecimiento holístico de los docentes en el contexto educativo con una visión a corto, mediano y largo plazo que contribuya a mejorar el proceso de enseñanza - aprendizaje en entornos virtuales, requiere de un efectivo uso de los recursos digitales con la finalidad de lograr un aprendizaje significativo (Granados-López \& García-Zuluaga, 2016).

La integración y usos de las tecnologías de la información en el proceso de enseñanza aprendizaje, es un reto actual cuyo cumplimiento requiere salir de la zona de confort que ha sido una herencia dada por la enseñanza tradicional, una vez que el docente tiene la predisposición de emprender un cambio educativo hemos logrado un $90 \%$ en innovar las prácticas áulicas cotidianas, el 10\% restante corresponde al espíritu indagador de cada docente, ya que en la actualidad tenemos todas la herramientas a un clic de distancia. 


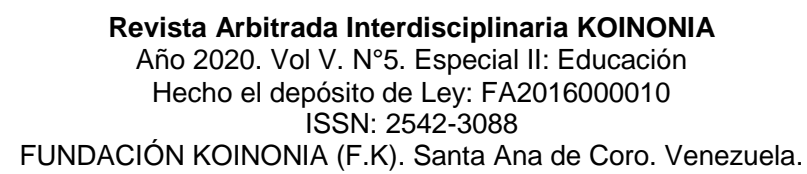

Ana Daniela Cabrera-Padrón; Darwin Gabriel García-Herrera; Juan Carlos Erazo-Álvarez Cecilia Ivonne Narváez-Zurita

Por otro lado, (Levis, 2011), señala las ventajas que tienen las redes educativas, en el proceso de fortalecimiento docente:

1. Favorecen al desarrollo de actividades en grupos afines por áreas de interés.

2. Propician la creación de estrategias dinámicas cooperativas y colaborativas en el campo del estudio y de la investigación.

3. Dan cabida al trabajo interdisciplinar que permite analizar estrategias desde diferentes áreas del aprendizaje.

4. Promueven el sentimiento de pertenencia hacia el grupo, ayudando a la implementación de redes de aprendizaje.

5. Permite la indagación de los conocimientos previos, que para Ausubel son ideas de anclaje que cimientan la base para nuevos aprendizajes.

6. Fomenta la generación colectiva de conocimiento.

Cabe señalar lo manifestado por (Abuín \& Mediavilla, 2008), quien habla del gran valor de la Web 2.0 en el ámbito de educación ya que los integrantes comparten conocimientos y experiencias que sirven de punto de partida para los demás, convirtiéndose estos sitios virtuales que permiten la interacción y comunicación, mediante el uso de la tecnología. Para (Abuín \& Mediavilla, 2008) entre los entornos virtuales más distintivos de la Web 2.0 tenemos:

Weblogs: Son sitios web que se utilizan como bitácoras virtuales, para la recopilación de artículos y textos de uno o más autores, esta puede ser organizada según las temáticas, fecha de creación del archivo y uso. Esta herramienta aplicada entre docentes es de mucha utilidad para compartir artículos científicos y de interés educativo.

Podcasts: Son grabaciones sonoras, que pueden ser enviadas por muchas plataformas sociales, se puede emplear como apoyo para transferir ideas principales, opiniones, comentarios e incluso instrucciones dentro del contexto educativo.

Videocasetes: Se manejan en formato MPEG 4 de video, es decir se trata de la integración entre el podcast (audio) y la imagen en movimiento (video). Es una herramienta muy útil principalmente si queremos realizar Storytelling. 
Revista Arbitrada Interdisciplinaria KOINONIA

Año 2020. Vol V. N5. Especial II: Educación

Hecho el depósito de Ley: FA2016000010

ISSN: 2542-3088

FUNDACIÓN KOINONIA (F.K). Santa Ana de Coro. Venezuela.

\section{Ana Daniela Cabrera-Padrón; Darwin Gabriel García-Herrera; Juan Carlos Erazo-Álvarez} Cecilia Ivonne Narváez-Zurita

Wikis: Es una herramienta digital que permite el trabajo colaborativo es decir permite el acceso de varios usuarios para la edición, creación y alimentación de documentos alojados en la web.

Redes Sociales: son espacios virtuales que permiten la interacción entre los usuarios de manera bidireccional, su principal característica es que se trata de un sistema abierto que se va nutriendo con lo que cada usuario sube a la plataforma. Estos espacios son muy importantes al momento de establecer vínculos afectivos, sociales y de negocios.

Esta infinidad de plataformas digitales con todo un abanico de posibilidades y beneficios son imprescindibles en el entorno virtual que estamos obligados a vivir, para conocer el uso y manejo de estas herramientas tecnológicas, no es necesario volver a las aulas de clase como dijimos en un principio podemos aprovechar los conocimientos y experiencias de otros docentes para integrar la tecnología en el proceso de enseñanza aprendizaje.

En cuanto a integrar la tecnología en el proceso de enseñanza aprendizaje, (Delgado \& Solano, 2009), manifiestan que, ser un docente intermediario en entornos virtuales, no quiere decir cambiar el espacio físico a un aula virtual, reemplazar los textos escolares por documentos electrónicos, sustituir la lluvia de ideas de la clase por foros virtuales; Ser un docente intermediario tiene un sentido más amplio, implica buscar y aplicar estrategias de aprendizaje, y utilizar recursos educativos que logren la construcción de conocimientos, cumpliendo con los objetivos planteados hasta llegar a la consolidación de un aprendizaje significativo.

Así mismo, (Delgado \& Solano, 2009), Sostienen que los docentes deben garantizar la calidad educativa en los entornos virtuales de aprendizaje, mediante la realización de las siguientes funciones:

1. Función de planeación. Es un tiempo en el que el docente reflexiona sobre el tiempo, metodología y evaluación, requiere de un proceso pedagógico continuo y metódico, este proceso se puede fortalecer con las experiencias de cada docente al trabajar de manera colaborativa. 


\section{Ana Daniela Cabrera-Padrón; Darwin Gabriel García-Herrera; Juan Carlos Erazo-Álvarez} Cecilia Ivonne Narváez-Zurita

2. Función comunicativa, permite que el estudiante conozca y se prepare para cada momento de la clase, manteniéndolo motivado y a la expectativa de cada paso del proceso de enseñanza aprendizaje.

3. Función evaluativa, este proceso es imprescindible en el proceso de enseñanza aprendizaje ya que permite indagar sobre el proceso, y tomar las respectivas decisiones.

4. Función de diseño en entornos virtuales de aprendizaje (EVA).

Este proceso se puede desarrollar en un entorno colaborativo en el que cada docente aporte sus conocimientos y experiencias dentro del proceso de planeación, aplicación y evaluación en el proceso de enseñanza aprendizaje.

\section{METODOLOGÍA}

Metodológicamente se trabajó con un enfoque mixto de prevalencia descriptiva, recopilandose datos cuantitativos, mediante encuesta e instrumento de 10 preguntas en escala Likert, validadas por el Alfa de Cronbach con resultado de 0,926, cualitativamente fue la entrevista, la técnica de recopilación y el guión de preguntas semi estructurada para la recepción de información por grupo focal, en una muestra de de 23 docentes y 3 directivos de la Unidad Educativa del Milenio Paiguara de la Parroquia San Juan del cantón Gualaceo - Ecuador, para la aplicación del grupo focal se desarrollo un banco de preguntas validadas por el método Delphi, que consistió en la validación del instrumento por expertos en la materia.

Se analizaron los datos cuantitativos en razón de la estadistica descriptiva y chi cuadrado de Pearson, cualitativamente en razón del análisis de contenido.

\section{RESULTADOS}

Al calcular los datos estadisticos descriptivos se manifiesta que el trabajo colaborativo es primordial para afianzar la práctica docente, por lo tanto la formación de redes de apoyo entre docentes es indispensable en el contexto educativo ya que permite el intercambio 
de conocimientos y experencias, además de identificar y resolver posibles conflictos a partir de un enfoque interdisciplinar.

\section{Tabla 1}

El trabajo colaborativo entre docentes nos permite trabajar coordinadamente y con un mismo fin que es mejorar la calidad educativa.

\begin{tabular}{ccccc}
\hline Valido & Frecuencia & Porcentaje & $\begin{array}{l}\text { Porcentaje } \\
\text { Valido }\end{array}$ & Porcentaje Total \\
\hline Muy de Acuerdo & 14 & 60,9 & 60,9 & 60,9 \\
De Acuerdo & 8 & 34,8 & 34,8 & 95,7 \\
Indiferente & 1 & 4,3 & 4,3 & 100,0 \\
Total & 23 & 100,0 & 100,0 & \\
\hline
\end{tabular}

Fuente: Elaboración propia

La tabla 1 revela que del total de los docentes encuestados, el 95\% considera que el trabajo colaborativo permite sincronizar la práctica docente en busca del mejoramiento de la calidad educativa, por el contrario el el 4,3\% es indiferente a este enunciado. Estos datos evidencian la importancia del trabajo colaborativo entre docentes para afianzar los conocimientos y experiencias en el proceso de enseñanza aprendizaje.

\section{Tabla 2}

La formación de redes de apoyo entre docentes es una práctica educativa interdisciplinar que permite fortalecer el dialogo entre los integrantes de la comunidad educativa.

\begin{tabular}{lcccc}
\hline \multicolumn{1}{c}{ Valido } & Frecuencia & Porcentaje & Porcentaje Valido & Porcentaje Total \\
\hline Muy de Acuerdo & 6 & 26,1 & 26,1 & 26,1 \\
De Acuerdo & 14 & 60,9 & 60,9 & 87,0 \\
Indiferente & 2 & 8,7 & 8,7 & 95,7 \\
En desacuerdo & 1 & 4,3 & 4,3 & 100,0 \\
Total & 23 & 100,0 & 100,0 & \\
\hline
\end{tabular}

Fuente: Elaboración propia. 
Revista Arbitrada Interdisciplinaria KOINONIA

Año 2020. Vol V. N5. Especial II: Educación

Hecho el depósito de Ley: FA2016000010

ISSN: 2542-3088

FUNDACIÓN KOINONIA (F.K). Santa Ana de Coro. Venezuela.

Ana Daniela Cabrera-Padrón; Darwin Gabriel García-Herrera; Juan Carlos Erazo-Álvarez Cecilia Ivonne Narváez-Zurita

La tabla 2 revela que del total de los docentes encuestados, el $87 \%$ considera que la formación de redes de apoyo entre los docentes fortalece el diálogo entre los integrantes de la comunidad educativa, el $8,7 \%$ es indiferente, mientras que el $4,3 \%$ esta en desacuerdo en este enunciado. Este enunciado revela que el intercambio de experiencias pueden afianzar la comunicación acertiva entre los docentes.

\section{Tabla 3.}

Tabulación cruzada entre las variables: El cambio de la educación presencial a la virtual requiere mayor compromiso por parte de los docentes y me gustaría formar parte de una red de apoyo entre docentes para mejorar mi práctica educativa.

\begin{tabular}{|c|c|c|c|c|c|c|}
\hline & & \multicolumn{4}{|c|}{$\begin{array}{l}\text { Me gustaría formar parte de una red de apoyo entre } \\
\text { docentes para mejorar mi práctica educativa. }\end{array}$} & \multirow[b]{2}{*}{ Total } \\
\hline & & $\begin{array}{c}\text { En total } \\
\text { desacuerdo }\end{array}$ & $\begin{array}{c}\text { Indiferent } \\
\mathrm{e}\end{array}$ & De Acuerdo & $\begin{array}{c}\text { Muy de } \\
\text { Acuerdo }\end{array}$ & \\
\hline \multirow{5}{*}{$\begin{array}{c}\text { El cambio } \\
\text { de la } \\
\text { educación } \\
\text { presencial a } \\
\text { la virtual } \\
\text { require } \\
\text { mayor } \\
\text { compromis } \\
\text { o por parte } \\
\text { de los } \\
\text { docentes. }\end{array}$} & En total desacuerdo & 0 & 1 & 0 & 0 & 1 \\
\hline & En desacuerdo & 1 & 0 & 0 & 1 & 2 \\
\hline & Indiferente & 0 & 1 & 0 & 0 & 1 \\
\hline & De Acuerdo & 0 & 1 & 4 & 1 & 6 \\
\hline & Muy de Acuerdo & 0 & 1 & 4 & 8 & 13 \\
\hline \multicolumn{2}{|r|}{ Total } & 1 & 4 & 8 & 10 & 23 \\
\hline \multicolumn{3}{|r|}{ Valor } & \multicolumn{2}{|c|}{ Df } & \multicolumn{2}{|c|}{$\begin{array}{l}\text { Sig. asintótica } \\
\text { (bilateral) }\end{array}$} \\
\hline \multicolumn{2}{|c|}{ Chi- cuadrado de Pearson } & $25,462^{a}$ & \multicolumn{2}{|c|}{12} & \multicolumn{2}{|c|}{0,013} \\
\hline \multicolumn{2}{|c|}{ Razón de verosimilitudes } & 18,309 & \multicolumn{2}{|c|}{12} & \multicolumn{2}{|l|}{0,107} \\
\hline \multicolumn{2}{|c|}{ Asociación lineal por lineal } & 6,697 & \multirow{2}{*}{\multicolumn{2}{|c|}{1}} & \multirow{2}{*}{\multicolumn{2}{|c|}{0,010}} \\
\hline \multicolumn{2}{|c|}{$\mathrm{N}$ de Casos Validos } & 23 & & & & \\
\hline
\end{tabular}

Fuente: Elaboración propia. 
Revista Arbitrada Interdisciplinaria KOINONIA

Año 2020. Vol V. №5. Especial II: Educación

Hecho el depósito de Ley: FA2016000010

ISSN: 2542-3088

FUNDACIÓN KOINONIA (F.K). Santa Ana de Coro. Venezuela.

Ana Daniela Cabrera-Padrón; Darwin Gabriel García-Herrera; Juan Carlos Erazo-Álvarez Cecilia Ivonne Narváez-Zurita

Las pruebas de chi-cuadrado nos revelan que existe relación entre el mayor compromiso por parte de los docentes dentro de los entornos virtuales y la alternativa de la formación de redes de apoyo para afianzar la práctica educativa, esto nos permitirá implementar de mejor manera nuestra propuesta.

Tabla 5.

Análisis de resultados cualitativos.

\begin{tabular}{|c|c|c|}
\hline $\begin{array}{l}\text { Unidad de } \\
\text { análisis }\end{array}$ & Categoría & Segmento \\
\hline \multirow[t]{5}{*}{$\begin{array}{l}\text { Interacción } \\
\text { entre docentes }\end{array}$} & $\begin{array}{l}\text { Intercambio de } \\
\text { conocimientos y } \\
\text { experiencias. }\end{array}$ & $\begin{array}{l}\text { El intercambio de experiencias vividas dentro de la práctica docente, } \\
\text { nos permite conocer distintas realidades, entornos, actividades y } \\
\text { acciones, consideradas desde una perspectiva diferente. } \\
\text { El intercambio de saberes nos lleva al análisis, la argumentación y la } \\
\text { criticidad permitiendo la retroalimentación de la práctica docente. }\end{array}$ \\
\hline & $\begin{array}{l}\text { Trabajo } \\
\text { colaborativo }\end{array}$ & $\begin{array}{l}\text { Se considera que trabajar colaborativamente mejora el proceso de } \\
\text { enseñanza aprendizaje ya que ayuda a precisar las ideas, mejorar } \\
\text { las estrategias de aprendizaje y la toma de decisiones. } \\
\text { Trabajar de forma interdisciplinaria la enseñanza va ser más eficaz y } \\
\text { eficiente. }\end{array}$ \\
\hline & Redes de apoyo & $\begin{array}{l}\text { Mediante redes de apoyo se puede intercambiar experiencias de } \\
\text { enseñanza y aprendizaje, se comparte las fortalezas y dificultades en } \\
\text { los procesos de enseñanza y al mismo tiempo se porponen o } \\
\text { plantean soluciones a los problemas académicos. }\end{array}$ \\
\hline & & $\begin{array}{l}\text { Con la formación de redes de apoyo se pueden mejorar aspectos } \\
\text { como: Estrategias metodologicas, manejo de recursos didácticos } \\
\text { digitales, atención a NEE, inclusión y atención a la diversidad,los } \\
\text { espacios de intercambio de experiencias docentes, formación } \\
\text { profesional, resultados de aprendizaje de los estudiantes, } \\
\text { convivencia entre los miembros de la comunidad educativa. }\end{array}$ \\
\hline & $\begin{array}{l}\text { Compromiso por } \\
\text { parte de las } \\
\text { autoridades. }\end{array}$ & $\begin{array}{l}\text { Dar apertura a la formación de redes de apoyo dentro de la } \\
\text { comunidad educativa y potenciar este proyecto buscando aliados } \\
\text { estratégicos dentro y fuera de la institución, que oferten el } \\
\text { mejoramiento profesional individual y colectivo. }\end{array}$ \\
\hline
\end{tabular}

Fuente: Elaboración propia 


\section{Ana Daniela Cabrera-Padrón; Darwin Gabriel García-Herrera; Juan Carlos Erazo-Álvarez} Cecilia Ivonne Narváez-Zurita

\section{Unidad de análisis y categorías}

\section{Intercambio de conocimientos y experiencias.}

El ritmo actual en el desarrollo y uso de la tecnologia, require una mayor capacitación por parte de los docentes, lamentablemente algunos docentes del sector público atribuyen su falta de capacitación al estado, manifestando que son los llamados a buscar los recursos y espacios de tiempo para capacitar a los docentes. Dejando a un lado estos infundados argumentos, podemos aprovechar el conocimiento y la experiencia de los docentes que trabajan dentro de una misma institución educativa para fortalecer la practica docente.

\section{Trabajo colaborativo}

Es una estrategia muy aplicada en diferentes campos de estudio para aunar esfuerzos y conseguir un objetiuvo en común. En el campo de la educación se emplea mucho en grupos de estudiantes para fomentar valores y dividir responsabilidades, en cuanto al trabajo colaborativo entre docentes, dentro del contexto educativo permite mejorar y potenciar el proceso de enseñanza aprendizaje ya que contempla un enfoque interdisciplinar que permite un analisis crítico de diferentes enfoque y puntos de vista que nutren la práctica docente.

\section{Redes de apoyo}

Las redes de apoyo son alineaciones organizadas que permiten trabajar de manera sincronizada, es decir es una estructura formal en donde su principal enfoque es el trabajo colaborativo, la principal caracteristica de las redes de apoyo es que permite intercambiar experiencias sobre: Estrategias metodologicas, manejo de recursos didácticos digitales, atención a NEE, inclusión, atención a la diversidad,entre otros permitiendo detectar falencias en estos procesos y fortalecerlos. 


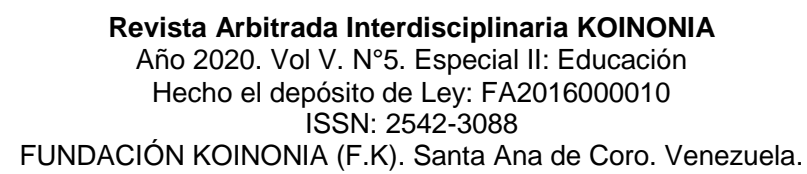

Ana Daniela Cabrera-Padrón; Darwin Gabriel García-Herrera; Juan Carlos Erazo-Álvarez Cecilia Ivonne Narváez-Zurita

\section{Compromiso por parte de las autoridades.}

La predispocición por parte de las autoridades y docentes para lograr una mejora en la calidad educativa es fundamental para lograr un cambio en el proceso de enseñanza aprendizaje, en los compromisos concensuados pora parte de las autoridades es dar apertura a la formación de redes de apoyo entre docentes y posteriormente fortalecer este proyecto con la participación de aliados estratégicos dentro y fuera de la unidad educativa.

En este sentido, (García-Pérez, et al., 2015), expresan que la importancia del trabajo colaborativo entre docentes, debe centrarse en lo que representa el enseñar y la importancia de aprender en el contexto actual, en este punto al realizar una autoevaluación se puede situar que se han logrado algunos objetivos como son compartir conocimientos, experiencias, herramientas y recursos para cubrir las necesidades detectadas, además de brindar respuestas situadas en el contexto de nuestra unidad educativa.

La investigación realizada sobre redes de apoyo y colaboración para la mejora de la educación inclusiva, en la que (Sánchez, et al., 2018), plantea en los resultados, que el proyecto ha permitido crear espacios de interacción y formar una red de aprendizaje dentro y fuera de los centros educativos participantes, logrando su principal objetivo que fue el desarrollo y mejora de la educación inclusiva.

Como lo manifiesta (Gordó-i-Aubarell, 2012), las redes de apoyo son nuevas versiones de estructuras educativas, cimentadas en interacción de sus participantes, con una sola finalidad dejar a un lado las individualidades para buscar soluciones comunitarias.

De la misma manera en la investigación sobre: fortaleciendo el trabajo colaborativo con las redes de apoyo de los establecimientos educativos, (Araos \& Álvarez, 2017), concluyerón que el compromiso y la implicación de los integrantes de la red permitieron el analisis y fortalecimiento de varios aspectos de la Educación Parvularia,como: Juegos, actividades lúdicas, bienestar, seguridad entre los más importantes, además el trabajo 
colaborativo permitieron plantear nuevos proyectos con distintos actores para mejorar la calidad en la educación.

Estas líneas de argumentación afirmaron la importancia del trabajo colaborativo y la formación de estructuras que permitan el intercambio de experiencias, además el análisis de los resultados nos motivó a contuniar con nuestra investigación: formar redes de apoyo para fortalecer la práctica docente.

\section{PROPUESTA}

La formación de redes de apoyo para fortalecer el proceso de enseñanza aprendizaje, se fundamentó en una trilogía de $3 \mathrm{Cs}$, compromiso, colaboración y cambio, estos escenarios son prioritarios y nos permitirá garantizar el propósito de esta investigación y optimizar el intercambio de conocimientos y experiencias por parte de los docentes dentro del contexto educativo, con el único objetivo, el fortalecimiento de la práctica docente.

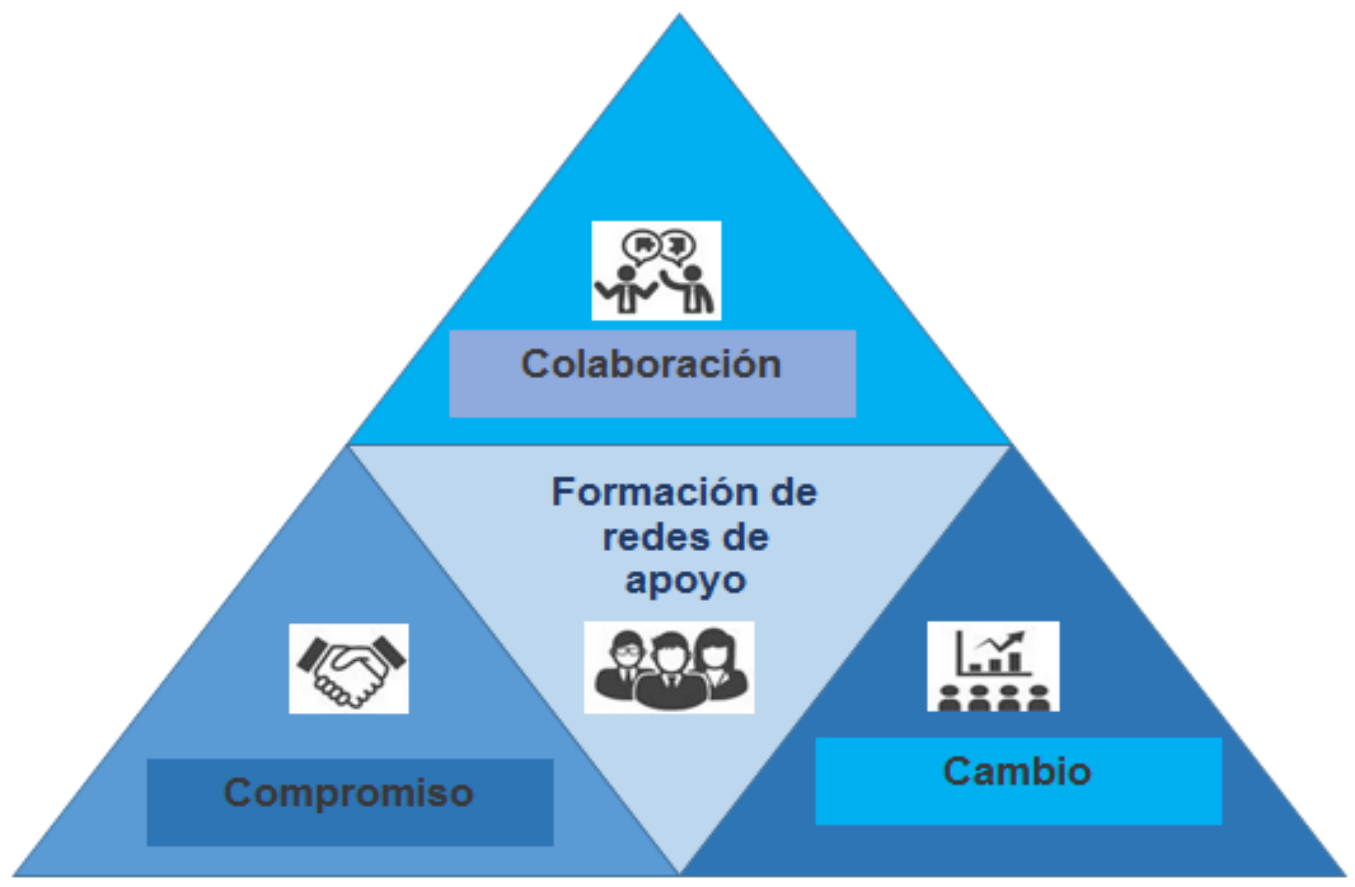

Figura 1. Trilogía 3Cs.

Fuente: Elaboración propia. 


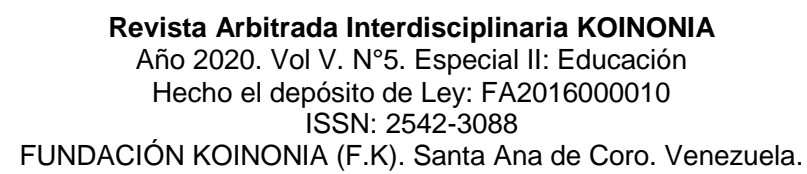

Ana Daniela Cabrera-Padrón; Darwin Gabriel García-Herrera; Juan Carlos Erazo-Álvarez Cecilia Ivonne Narváez-Zurita

Compromiso: Se impartió una capacitación a los docentes de la unidad educativa del milenio Paiguara sobre la formación de redes de apoyo entre docentes, dando a conocer la importancia del trabajo colaborativo para potenciar la práctica docente. De este modo se logro el compromiso de los miembros de la comunidad educativa para integrar la primera red de apoyo entre docentes en esta prestigiosa institución. Al finalizar este primer escenario se desarrolló una Autoevaluación dando respuesta a estas interrogantes:

a) ¿La práctica docente necesita una constante capacitación?

b) ¿Me interesa formar parte de esta red de trabajo colaborativo?

c) ¿Estoy dispuesto a trabajar colaborativamente?

Con este documento se consolido un compromiso por escrito por parte de los actores de esta investigación.

Colaboración: En este escenario se contó con el apoyo de directivos y docentes capacitados en diferentes aspectos como: Estrategias metodologicas, manejo de recursos didácticos digitales, atención a NEE, inclusión y atención a la diversidad, con la finalidad de garantizar la calidad de información y experiencias trabajadas colaborativamente. Esta etapa culminó con una co evaluación en la que se aplicó el siguiente cuestionario.

a) ¿Qué aprendizajes nos dejó esta etapa del proyecto?

b) ¿De los temas expuestos, cual fue el más interesante?

c) ¿Qué aspectos se requieren profundizar mas?

d) ¿A que se compromete como miembro de la red de apoyo?

e) ¿De los temas expuestos, con cual no estuvo de acuerdo?

Esto nos permitío realizar un análisis de las debilidades y fortalezas en esta etapa del proyecto. 


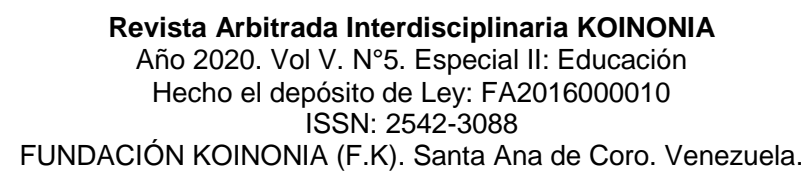

Ana Daniela Cabrera-Padrón; Darwin Gabriel García-Herrera; Juan Carlos Erazo-Álvarez Cecilia Ivonne Narváez-Zurita

Cambio: La evaluación dentro del contexto educativo es fundamental para el mejoramiento de la práctica docente ya que permite detectar durante el proceso los aspectos a mejorar y las potencialidades, dando lugar a una retroalimentación que nos lleva al cambio, para lograr esto es impresindible que el docente este abierto al proceso de hetero evaluación por parte de los integrantes de la red de apoyo,mediante la aplicación de una ficha para la observacion aúlica. Esto sin duda ayudará a alcanzar la calidad y calidez que tanto se predica.

\section{CONCLUSIONES}

La práctica docente es un cúmulo de experiencias de aula muy complejas, lamentablemente en muchas ocasiones nos encontramos con docentes que se cierran ante sugerencias o simplemente no aceptan la retroalimentación luego del acompañamiento por parte de las autoridades, es por ello que consideramos que la educación tradicional se mantendrá si no empezamos por uno mismo, aquí radica la importancia de la autoevaluación en la práctica docente, cabe concluir que este proceso nos permite identificar nuestras fortalezas y debilidades, este debe ser el punto de partida en la búsqueda de la calidad educativa.

Una vez echa esta precisión, es importante señalar que para mejorar la calidad en el proceso de enseñanza - aprendizaje dentro de un contexto educativo, todos sus integrantes deben estar comprometidos con la institución educativa, esto se logra con la debida motivación y consensos por parte de las autoridades del establecimiento, además deben construir participativamente objetivos a corto, mediano y largo plazo, solo de este modo se podrán aunar esfuerzos, proyectar propuestas y trabajar colaborativamente para alcanzar la meta común planteada.

Continuando con esta retrospectiva, voy a referirme brevemente a las ventajas de la implementación de este proyecto en el contexto educativo, que además de llegar al objetivo plateado que fue fortalecer el proceso de enseñanza aprendizaje, permitió la creación de redes de apoyo entre docentes, dentro de las cuales se destaca el trabajo 
colaborativo, la interacción en el intercambio de conocimientos, ideas, y experiencias y lo más importante se plantó la semilla de la investigación, como parte fundamental en la actualización dentro del ámbito educativo.

Todo lo dicho hasta ahora explica porque la premisa de nuestra propuesta fue fortalecer el proceso de enseñanza aprendizaje, mediante la implementación de redes de apoyo. Esto se logró con la aplicación de la trilogía 3Cs, denominada así por los tres escenarios: Compromiso en la formación de la red de apoyo, colaboración o trabajo en equipo y cambio en la práctica docente, estos tres espacios nos permitieron construir una estructura sólida, basada en el compromiso y la comunicación asertiva, punto importante en el trabajo colaborativo ya que un equipo sin jugadores no puede ganar el campeonato.

\section{FINANCIAMIENTO}

No monetario

\section{AGRADECIMIENTO}

A la Unidad Educativa del Milenio Paiguara de la Parroquia San Juan del cantón Gualaceo - Ecuador; por apoyar el desarrollo de la investigación.

\section{REFERENCIAS CONSULTADAS}

Abuín, N., \& Mediavilla, C. (2008). De la Web a la Web 3.0: un análisis comparativo entre la Web, la Web Social y la Web Semántica. Comunicación [Recurso electrónico]: Memoria, Historia y Modelos, 1-11.

Aparicio-Molina, C, \& Sepúlveda-López, F. (2019). Trabajo colaborativo docente: nuevas perspectivas para el desarrollo docente. [Trabalho colaborativo docente: novas perspectivas para 0 desenvolvimento docente]. Psicologia Escolar $e$ Educacional, 23, e187926. Epub December 09, 2019.https://dx.doi.org/10.1590/2175-35392019017926 
Revista Arbitrada Interdisciplinaria KOINONIA

Año 2020. Vol V. N5. Especial II: Educación

Hecho el depósito de Ley: FA2016000010

ISSN: 2542-3088

FUNDACIÓN KOINONIA (F.K). Santa Ana de Coro. Venezuela.

Ana Daniela Cabrera-Padrón; Darwin Gabriel García-Herrera; Juan Carlos Erazo-Álvarez Cecilia Ivonne Narváez-Zurita

Araos, M. J., \& Álvarez, C. (2017). Fortaleciendo el trabajo colaborativo con las redes de apoyo de los establecimientos educativos. Universidad Alberto Hurtado, 1-13.

Cochran-smith, M., \& Lytle, S. L. (2006). Troubling images of teaching in No Child Left Behind. Harvard Educational Review, 668-726 DOI: 10.17763/haer.76.4.56v8881368215714.

Delgado, M., \& Solano, A. (2009). Estrategias didácticas creativas en entornos virtuales para el aprendizaje. Actualidades Investigativas en Educación, 19(2),1-21.

Feixas, M. (2004). La influencia de factores personales, institucionales y contextuales en la trayectoria y el desarrollo docente de los profesores universitarios. Educar, 33, 31-59

Fullan, M., \& Hargreaves, A. (1997). La escuela que queremos [The school we want]. Argentina: AMORRORTU.

García-Pérez, Y, Herrera Rodríguez, J, García Valero, M, \& Guevara Fernández, G. (2015). El trabajo colaborativo y su influencia en el desarrollo de la cultura profesional docente. [Collaborative work and its influence on the development of teaching professional culture]. Gaceta Médica Espirituana, 17(1), 60-67.

Gordó-i-Aubarell, G. (2012). Del centro educativo a la red educativa. Planes educativos de entorno, una organización en red. [From the educational center to the educational network. Environmental educational plans, a network organization]. En Redes Educativas: La educación en la sociedad del conocimiento. (1-11), Sevilla, España: Universidad de Sevilla. Grupo de Investigación Didáctica.

Granados-López, H, \& García-Zuluaga, C. (2016). El modelo de aprendizaje experiencial como alternativa para mejorar el proceso de aprendizaje en el aula. [The experiential learning model as an alternative to improve the learning process in the classroom]. Ánfora, 23(41),37-54.

Levis, Diego (2011). Redes educativas 2.1 Medios sociales, entornos colaborativos y procesos de enseñanza y aprendizaje. [Educational networks 2.1 Social media, collaborative environments and teaching and learning processes]. RUSC. Universities and Knowledge Society Journal, 8(1),7-24.

Ministerio de Educación del Ecuador (2019). Redes de aprendizaje [Learning networks]. Recuperado de https://n9.cl/wswl 
Revista Arbitrada Interdisciplinaria KOINONIA

Año 2020. Vol V. N5. Especial II: Educación

Hecho el depósito de Ley: FA2016000010

ISSN: 2542-3088

FUNDACIÓN KOINONIA (F.K). Santa Ana de Coro. Venezuela.

Ana Daniela Cabrera-Padrón; Darwin Gabriel García-Herrera; Juan Carlos Erazo-Álvarez Cecilia Ivonne Narváez-Zurita

Montse-Guitert, I, \& Ferran-Giménez, C. (2000). Trabajo cooperativo en entornos virtuales de aprendizaje. Aprender en la virtualidad. En Aprender en la virtualidad/coord. por Josep María Duart Montoliu, Albert Sangrá Morer. Gedisa : Universitat Oberta de Catalunya. 113-134.

Muijs, D., \& Rumyantseva, N. (2014). Coopetition in education: Collaborating in a competitive environment. Journal of Educational Change, 15, 1-18 https://doi.org/10.1007/s10833-013-9223-8

Murillo, F. J., \& Krichesky, G. J. (2014). Mejora de la Escuela: Medio siglo de lecciones aprendidas. [Improvement: A half-century of learned lessons]. REICE. Revista Iberoamericana sobre Calidad, Eficacia y Cambio en Educación, 59-102 URL: https://n9.cl/yn9p.

Podestá, P. (2014). El trabajo colaborativo entre docentes: experiencias en la Especialización Docente Superior en Educación y TIC. Congreso Iberoamericano de Ciencia, Tecnología, Innovación y Educación. Congreso llevado a cabo en Buenos Aires, Argentina.

Ponce-Ceballos, S, García-Cabrero, B, Islas-Cervantes, D, Martínez-Soto, Y, \& SernaRodríguez, A. (2018). De la tutoría a la mentoría. Reflexiones en torno a la diversidad del trabajo docente. [From Tutoring to Mentoring. Reflections on the Diversity of Teaching Functions]. Páginas de Educación, 11(2), 215235. https://dx.doi.org/10.22235/pe.v11i2.1635

Ramírez-Iñiguez, A. (2017). La educación con sentido comunitario: reflexiones en torno a la formación del profesorado. [Community-based education: reflections on teacher education]. Educación, 26(51),

$79-$ 94. https://dx.doi.org/10.18800/educacion.201702.004

Sánchez, A., De-Haro-Rodríguez, R, \& Azorín-Abellán, C. (2018). Redes De Apoyo Y Colaboración Para La Mejora De La Educación Inclusiva. Profesorado, Revista de Currículum y Formación del Profesorado, 22(2); 1-21

Sánchez, P. A., Rodríguez, R. D., \& Abellán, C. M. (2018). Redes de apoyo y colaboración para la mejora de la educación inclusiva. [Support and Collaboration Networks for Improving Inclusive Education]. Profesorado, Revista de Currículum y Formación del Profesorado, 22(2); 30-49 
Revista Arbitrada Interdisciplinaria KOINONIA

Año 2020. Vol V. N5. Especial II: Educación

Hecho el depósito de Ley: FA2016000010

ISSN: 2542-3088

FUNDACIÓN KOINONIA (F.K). Santa Ana de Coro. Venezuela.

Ana Daniela Cabrera-Padrón; Darwin Gabriel García-Herrera; Juan Carlos Erazo-Álvarez Cecilia Ivonne Narváez-Zurita

Stoll, L., Bolam, R., Mcmahon, A., Wallace, M., \& Thomas, S. (2006). Professional Learning Communities: A Review of the Literature. Educ Change 7, 221-258. https://doi.org/10.1007/s10833-006-0001-8

Vaillant, D. (2016). Trabajo colaborativo y nuevos escenarios para el desarrollo profesional docente [Collaborative work and new scenarios for teacher professional development]. Revista Docencia, 5-13.

(C2020 por los autores. Este artículo es de acceso abierto y distribuido según los términos y condiciones de la licencia Creative Commons Atribución-NoComercial-Compartirlgual 4.0 Internacional (CC BY-NC-SA 4.0)

(https://creativecommons.org/licenses/by-nc-sa/4.0/). 
Revista Arbitrada Interdisciplinaria KOINONIA

Año 2020. Vol V. N5. Especial II: Educación

Hecho el depósito de Ley: FA2016000010

ISSN: 2542-3088

FUNDACIÓN KOINONIA (F.K). Santa Ana de Coro. Venezuela.

Ana Daniela Cabrera-Padrón; Darwin Gabriel García-Herrera; Juan Carlos Erazo-Álvarez Cecilia Ivonne Narváez-Zurita

\section{ANEXOS:}

\section{Anexo1.}

Ficha de observación áulica para el fortalecimiento de la práctica docente.

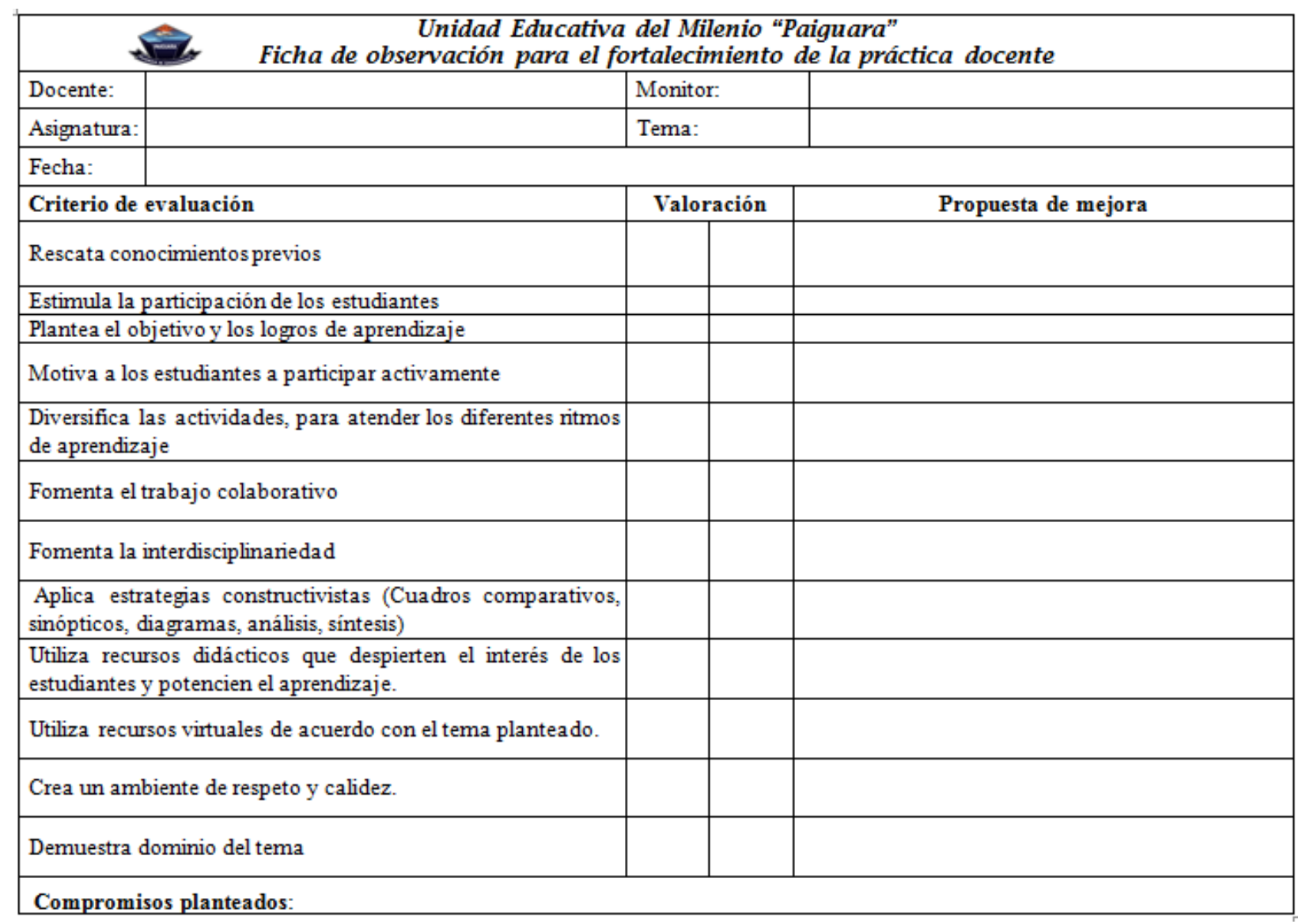

